\title{
Stochastic algorithms for discontinuous multiplicative white noise
}

\author{
R. Perez-Carrasco and J. M. Sancho \\ Departament d'Estructura i Constituents de la Matèria, Facultat de Física, Universitat de Barcelona, Diagonal 647, E-08028 Barcelona, \\ Spain
}

(Received 10 December 2009; published 22 March 2010)

\begin{abstract}
Stochastic differential equations with multiplicative noise need a mathematical prescription due to different interpretations of the stochastic integral. This fact implies specific algorithms to perform numerical integrations or simulations of the stochastic trajectories. Moreover, if the multiplicative noise function is not continuous then the standard algorithms cannot be used. We present an explicit algorithm to avoid this problem and we apply it to a well controlled example. Finally, we discuss on the existence of higher-order algorithms for this specific situation.
\end{abstract}

DOI: 10.1103/PhysRevE.81.032104

PACS number(s): 05.40.-a, 02.70.-c

Stochastic, ordinary, or partial differential equations are very common tools in far from equilibrium systems in physics or in other disciplines where stochastic fluctuations are necessary for an appropriate description of the phenomenology involved $[1,2]$. In the most interesting problems these equations cannot be solved analytically and stochastic numerical (simulation) algorithms have to be used to extract explicit results $[3,4]$.

Most problems involve the dynamics of a variable $x$ which obeys the Langevin stochastic equation

$$
\dot{x}=f(x)+g(x) \xi(t),
$$

where $\xi(t)$ is a Gaussian white noise with zero mean and covariation

$$
\left\langle\xi(t) \xi\left(t^{\prime}\right)\right\rangle=2 \delta\left(t-t^{\prime}\right),
$$

where we have reduced the complexity to a minimum. Although the meaning of Eqs. (1) and (2) has no mathematical problem when $g(x)=$ const (additive noise), important difficulties appear when $g(x)$ is a function of $x$. In this last case, the above Eq. (1) is mathematically not well-defined and different interpretations are possible. Two of them have been extensively studied: Itô and Stratonovich [5-7]. The former is popular between mathematicians because its nonanticipating property and the latter among applied researchers due to the fact that the standard rules of calculus can be used. The interpretations imply different Fokker-Planck equations for the probability distribution $p(x, t)$. So a natural question arises: which is the correct interpretation for a problem defined by Eqs. (1) and (2)? The answer is not straightforward and it does depend on the way these equations were derived. If they were obtained from a master equation a good choice is the Itô interpretation. If the origin is a Newton equation where the inertia (mass) has been discarded then Itô is also the choice. Nevertheless, if the noise $\xi(t)$ represents an approximation of a realistic process (nonwhite or colored), then Stratonovich is the answer. More elaborated arguments can be found in Ref. [8].

Let us summarize the mathematical origin of the interpretations. A formal integration of Eq. (1) from a time $t$ to $t+\Delta t$ is

$$
x(t+\Delta t)=x(t)+\int_{t}^{t+\Delta t} f\left[x\left(t^{\prime}\right)\right] d t^{\prime}+\int_{t}^{t+\Delta t} g\left[x\left(t^{\prime}\right)\right] \xi\left(t^{\prime}\right) d t^{\prime}
$$

As usual, one can Taylor expand $f\left[x\left(t^{\prime}\right)\right]$ and $g\left[x\left(t^{\prime}\right)\right]$ around $x(t)$ arriving to a simple algorithm to get $x(t+\Delta t)$ from the knowledge of $x(t)$. Then the first integral can be substituted by

$$
\int_{t}^{t+\Delta t} f\left[x\left(t^{\prime}\right)\right] d t^{\prime} \approx f[x(t)] \Delta t,
$$

which is the lowest contribution in $\Delta t$. Nevertheless, due to the very special character of the white noise the second integral in Eq. (3) is not univocally defined even to the lowest order in $\Delta t$.

There is a rich literature on the mathematical properties of stochastic integrals and how they are related with the properties of Gaussian white noise and Wiener random processes. A very recent work addresses this matter focusing on the use of the continuous-time random walk (CTRW) [5-7].

It is our statement that any proposed algorithm should be a direct outcome of the corresponding interpretation of the stochastic integral. Let us review now the main characteristics of the most used interpretations.

The Itô prescription is very simple. The second integral in Eq. (3) is evaluated as

$$
\int_{t}^{t+\Delta t} g\left[x\left(t^{\prime}\right)\right] \xi\left(t^{\prime}\right) d t^{\prime} \approx g[x(t)] \chi(t),
$$

where $\chi(t)$ is the Wiener increment (Gaussian process) defined as [6]

$$
\chi(t)=\int_{t}^{t+\Delta t} \xi\left(t^{\prime}\right) d t^{\prime}
$$

with zero mean value and second moment

$$
\left\langle\chi(t)^{2}\right\rangle=2 \Delta t
$$

The Wiener process can be related with the CTRW [7,9]. It is worth to remark here that the non-anticipating property of the Itô integral Eq. (5) fulfills the causality principle. 
Using the above results, the lowest order algorithm (Euler-Maruyama) $[4,10]$ for the Itô case is

$$
x(t+\Delta t)=x(t)+f[x(t)] \Delta t+g[x(t)] \chi(t)+\mathcal{O}\left(\Delta t^{3 / 2}\right),
$$

where the stochastic generation of $\chi(t)$ is a well controlled matter [2].

The Stratonovich prescription is $[5,6]$

$$
\int_{t}^{t+\Delta t} g\left[x\left(t^{\prime}\right)\right] \xi\left(t^{\prime}\right) d t^{\prime} \approx g\left[\frac{x(t)+x(t+\Delta t)}{2}\right] \chi(t) .
$$

Nevertheless, the above expression [Eq. (9)] drives to an implicit algorithm. The standard and simple procedure to avoid the implicit term and which had been used so far, is based in the assumption of continuity for the multiplicative function $g[x(t)]$, and the use of Eq. (8),

$$
g\left[\frac{x(t)+x(t+\Delta t)}{2}\right] \sim g[x(t)]+\frac{g^{\prime}[x(t)] g[x(t)]}{2} \chi(t),
$$

which drives to the lowest order Stratonovich algorithm

$$
\begin{aligned}
x(t+\Delta t)= & x(t)+f[x(t)] \Delta t+g[x(t)] \chi(t) \\
& +\frac{g^{\prime}[x(t)] g[x(t)]}{2} \chi(t)^{2}+\mathcal{O}\left(\Delta t^{3 / 2}\right),
\end{aligned}
$$

which is also called the Milstein scheme [11,12].

Moreover, it is well-known [6] that the Stratonovich interpretation of Eqs. (1) and (2) can be transformed in the Itô interpretation as

$$
\dot{x}=f(x)+\frac{1}{2} g^{\prime}(x) g(x)+g(x) \xi(t) .
$$

Accordingly, this equation can be numerically integrated with the Itô algorithm [Eq. (8)]. Thus, why bother about Stratonovich algorithm? Well, an important difficulty appears here, which is the main concern of this communication. If the function $g(x)$ is not continuous, neither the transformation in Eq. (12) nor the above algorithm [Eq. (11)] can be used. This is also extended to more elaborated algorithms [11-15]. But, what is the origin of this discontinuity? Piecewise ratchet potentials have become a very useful tool in the dynamical description of molecular machines and nonequilibrium systems $[16,17]$. If we assume that a part of this potential is a random process, then we will have discontinuous functions for $f(x)$ and $g(x)$. If we take now for simplicity that this random part can be approximated by a Gaussian white noise, then we arrive to the situation of our interest: a Langevin equation with a discontinuous multiplicative white noise function.

As we cannot make use of a nondefined $g^{\prime}(x)$ we can check alternative algorithms for the Stratonovich case that do not use the derivative of this function. They are based on predictor-corrector schemes [18], using the Itô algorithm [Eq. (8)] as the predictor step. The explicit algorithm is

$$
x_{\mathrm{I}}(t+\Delta t)=x(t)+f[x(t)] \Delta t+g[x(t)] \chi(t),
$$

$$
\begin{aligned}
x(t+\Delta t)= & x(t)+f[x(t)] \Delta t+g\left[\frac{x(t)+x_{\mathrm{I}}(t+\Delta t)}{2}\right] \chi(t) \\
& +\mathcal{O}\left(\Delta t^{3 / 2}\right) .
\end{aligned}
$$

Here we will denote it as algorithm I, which is compatible with the stochastic Stratonovich interpretation.

It is worth to relate algorithm I to the widely used Heun algorithm $[2,3,14,18]$, which substitutes the corrector step in [Eq. (13)] obtaining

$$
\begin{aligned}
x(t+\Delta t)= & x(t)+f[x(t)] \Delta t+\frac{g[x(t)]+g\left[x_{\mathrm{I}}(t+\Delta t)\right]}{2} \chi(t) \\
& +\mathcal{O}\left(\Delta t^{3 / 2}\right)
\end{aligned}
$$

The third term on the right-hand side of Eq. (14) implies another prescription for the Stratonovich stochastic integral [Eq. (9)], but in the case of a continuous $g(x)$ this algorithm is compatible with Eqs. (10) and (11) working quite well in this situation $[2,14,18]$.

We will see below in an example that both algorithms [Eqs. (13) and (14)] converge to solutions different from analytical ones with important systematic errors.

One can conclude that the intrinsic difficulty of the Stratonovich interpretation is to find an appropriate approximation for the implicit term in the stochastic integral. In order to avoid this problem one can try to make use of a not wellknown theorem which states that the Stratonovich prescription is the limit of a nonwhite (colored) noise when its covariation time goes to zero [19]. Although the theorem was proved for a multiplicative function $g(x)$ with continuous first derivative, we will explore numerically if this theorem also works for discontinuous functions.

We proceed by simulating Eq. (1) with a colored noise such as the Ornstein-Uhlenbeck process, $\xi_{\mathrm{OU}}(t)$, which follows the Langevin equation

$$
\tau \dot{\xi}_{\mathrm{OU}}(t)=-\xi_{\mathrm{OU}}(t)+\xi(t),
$$

and where $\xi(t)$ is a white noise [Eq. (2)]. The white noise limit of $\xi_{\mathrm{OU}}(t) \rightarrow \xi(t)$ is achieved for $\tau \rightarrow 0$.

A first order algorithm for the system in Eq. (1) with $\xi_{\mathrm{OU}}$ [Eq. (15)] is standard [11], and it reads

$$
\begin{gathered}
x(t+\Delta t)=x(t)+f[x(t)] \Delta t+g[x(t)] \xi_{\mathrm{OU}}(t) \Delta t+\mathcal{O}\left(\Delta t^{3 / 2}\right), \\
\xi_{\mathrm{OU}}(t+\Delta t)=\xi_{\mathrm{OU}}(t)\left(1-\frac{\Delta t}{\tau}\right)+\frac{\chi(t)}{\tau}+\mathcal{O}\left(\Delta t^{3 / 2}\right) .
\end{gathered}
$$

We will call it algorithm II and numerical results will be presented below. A value of $\tau$ smaller than any other characteristic time of the problem but much larger than $\Delta t$ has to be used. The last step in Eq. (16) can be substituted by other more refined methods to mimic exactly the OrnsteinUlenbeck process $[13,14,20]$. Moreover, the methods presented in Refs. $[13,14]$ do not have the restriction $\Delta t \ll \tau$. Algorithm II is our proposal to simulate stochastic trajectories for a discontinuous multiplicative white noise in the Stratonovich prescription. In order to validate the new algorithm II [Eq. (16)] we need an example with an exact theo- 
retical prediction to check its results and to make a comparison between different algorithms.

Our benchmark is a system that consists of an ensemble of noninteracting Brownian particles in a one dimensional container of length $L$ with two different temperatures in each half side. The Langevin equation is

$$
\dot{x}=g(x) \xi(t),
$$

where $g(x)$ is the temperature profile

$$
\left.g^{2}(x)\right|_{x<L / 2}=T_{a},\left.\quad g^{2}(x)\right|_{x>L / 2}=T_{b}, \quad T_{a}>T_{b} .
$$

A continuous representation of this function is

$$
g^{2}(x)=\frac{T_{b}+T_{a}+\left(T_{b}-T_{a}\right) \tanh [(x-L / 2) / \alpha]}{2},
$$

where the above limiting values appear for $\alpha \rightarrow 0$. The variable $x$ is in the domain $(0, L)$ with reflecting boundaries at $x=0$ and $x=L$.

The corresponding Fokker-Planck equations for the two interpretations are [6]

$$
\begin{gathered}
\frac{\partial p(x, t)}{\partial t}=\frac{\partial^{2}}{\partial x^{2}} g^{2}(x) p(x, t), \quad(\text { Itô }), \\
\frac{\partial p(x, t)}{\partial t}=\frac{\partial}{\partial x} g(x) \frac{\partial}{\partial x} g(x) p(x, t), \quad \text { (Strat.). }
\end{gathered}
$$

The steady solutions are constant profiles in each half side,

$$
p_{\text {Itô }}(x) \sim \frac{1}{g^{2}(x)}, \quad p_{\text {Strat. }}(x) \sim \frac{1}{g(x)},
$$

and from the numerical simulations we will analyze the ratios $R=p_{b} / p_{a}$,

$$
R_{\mathrm{Itô}}=\frac{T_{a}}{T_{b}}, \quad R_{\text {Strat. }}=\sqrt{\frac{T_{a}}{T_{b}}},
$$

where $p_{a}=p(x<L / 2)$ and $p_{b}=p(x>L / 2)$.

In Fig. 1 we present the numerical results for the different algorithms. We have performed very large time and ensemble averages to reduce the statistical errors and to enlighten the systematic errors. For the Itô algorithm [Eq. (8)] the agreement theory simulation is perfect. Algorithm II [Eq. (16)], for the Stratonovich interpretation, exhibits the same level of accuracy than the Itô algorithm, even for a not too small $\tau$. Thus the agreement with the theoretical prediction is well controlled now. In contrast, algorithm I [Eq. (13)] for the Stratonovich interpretation, involves important systematic numerical errors: $9 \%$ for $T_{a} / T_{b}=16$ and $44 \%$ for $T_{a} / T_{b}=64$. This constant drift from the exact solution is also present for the Heun algorithm [Eq. (14)], obtaining similar systematic numerical errors.

Moreover, for algorithm II we have found that the use of a colored noise whose time increments are correlated needs a careful attention if a reflecting boundary is present. When a particle is reflected at the boundary we need to change the sign of the colored noise (velocity) $\xi_{\mathrm{OU}}(t+\Delta t)$ in the next integration step. In Fig. 2 we present evidences of this phenomenon. If we do not invert the colored noise when a par-

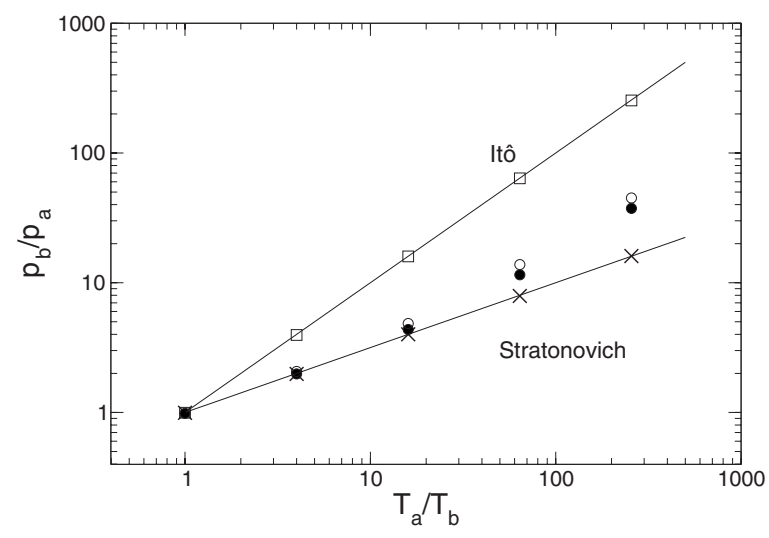

FIG. 1. Theoretical predictions (lines) and numerical simulation (symbols) results for different algorithms and stochastic interpretations in a log-log scale. Itô algorithm [Eq. (8)] (squares), Stratonovich algorithm I [Eq. (13)] (full circles), Heun algorithm [Eq. (14)] (circles), and Stratonovich algorithm II (Eq. (16)) with $\tau=10^{-3}$ (crosses). Parameters: $L=1, \Delta t=10^{-5}, n>10^{7}$ time steps, and $N=100$ trajectories.

ticle hits the wall, we found an anomalous density of particles near the reflecting walls (circles).

Higher-order algorithms make use of the derivatives of $f(x)$ and $g(x)$. Consequently, if these functions are not continuous, we cannot expect a much better algorithm than a Heun approximation for improving the deterministic term

$$
\int_{t}^{t+\Delta t} f\left[x\left(t^{\prime}\right)\right] d t^{\prime} \approx \frac{f[x(t)]+f\left[x_{\mathrm{I}}(t+\Delta t)\right]}{2} \Delta t,
$$

which can be incorporated either in Eq. (8) complemented with the predictor step or directly in Eq. (16).

Summarizing we have presented a lowest order algorithm [Eq. (16)] to simulate multiplicative white noise Langevin equations in the Stratonovich interpretation when function

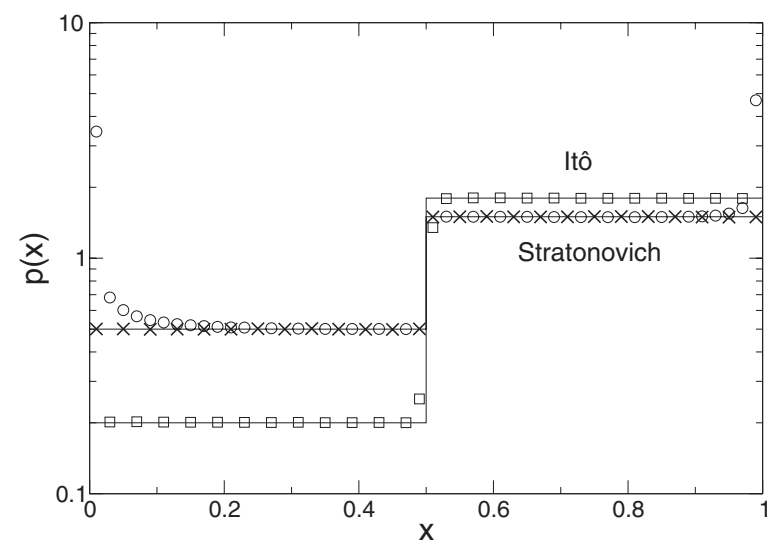

FIG. 2. Theoretical steady probability densities (lines). Itô algorithm (squares), Stratonovich algorithm II without noise inversion at the reflecting boundaries (circles) but adjusted to the bulk density and Stratonovich algorithm II with noise inversion (crosses). $T_{a} / T_{b}=9$. Other parameters as in previous figure. 
$g(x)$ is not continuous. We have shown that for this specific case the standard algorithms used commonly present a systematic drift, which is due to a biased estimation of the stochastic integral in this interpretation.

The proposed algorithm uses a colored noise with a small time scale and it is based in a mathematical theorem [19]. Nevertheless the algorithm goes beyond the conditions of the mathematical proof of this theorem. We expect that this Brief Report will stimulate mathematical approaches to establish rigorously these numerical results.
Finally as a by-product, we have shown that when the problem involves reflecting boundary conditions, algorithm II [Eq. (16)] has to include the inversion of the colored noise at the boundaries.

This work is supported by Ministerio de Ciencia e Innovación (Spain) through project No. FIS2009-13360 and FPU-AP2007-00987 (R.P.C.) and also by Generalitat de Catalunya through project No. 2009SGR14. Fruitful comments by M. Ibañes and R. Toral are also acknowledged.
[1] W. Horsthemke and R. Levever, Noise-Induced Transitions (Springer, Berlin, 1984).

[2] J. García-Ojalvo and J. M. Sancho, Noise in Spatially Extended Systems (Springer-Verlag, New York, 1999).

[3] T. C. Gard, Introduction to stochastic differential equations (Marcel Dekker, New York, 1987).

[4] P. E. Kloeden and E. Platen, Numerical Solution of Stochastic Differential Equations (Springer-Verlag, Berlin, 1999).

[5] R. L. Stratonovich, Topics in the Theory of Random Noise, Vols. 1 and 2 (Gordon and Breach, New York, 1967).

[6] C. W. Gardiner, Handbook of Stochastic Methods, 2nd ed, Springer Series in Physics Vol. 13 (Springer, Berlin, 1989).

[7] G. Germano, M. Politi, E. Scalas, and R. L. Schilling, Phys. Rev. E 79, 066102 (2009).

[8] R. Kupferman, G. A. Pavliotis, and A. M. Stuart, Phys. Rev. E 70, 036120 (2004).

[9] B. K. Øksendal, Stochastic Differential Equations: An Introduction with Applications (Springer, Berlin, 1985).

[10] G. Maruyama, Rend. Circ. Mat. Palermo 4, 48 (1955).
[11] J. M. Sancho, M. San Miguel, S. L. Katz, and J. D. Gunton, Phys. Rev. A 26, 1589 (1982).

[12] G. N. Milstein, Numerical Integration of Stochastic Differential Equations, Mathematics and Its Applications (Kluwer Academic, Norwell, MA, 1995).

[13] R. Mannella and V. Palleschi, Phys. Rev. A 40, 3381 (1989).

[14] M. San Miguel and R. Toral, Instabilities and Nonequilibrium Structures VI, edited by E. Tirapegui, J. Martínez, and R. Tiemann (Kluwer Academic, Norwell, MA, 2000), pp. 35-130,.

[15] Ch. Kim, E. K. Lee, and P. Talkner, Phys. Rev. E 73, 026101 (2006).

[16] F. Jülicher, A. Ajdari, and J. Prost, Rev. Mod. Phys. 69, 1269 (1997).

[17] P. Reimann, Phys. Rep. 361, 57 (2002).

[18] F. Sagués, J. M. Sancho, and J. García-Ojalvo, Rev. Mod. Phys. 79, 829 (2007).

[19] E. Wong and M. Zakai, Ann. Math. Stat. 36, 1560 (1965).

[20] R. F. Fox, I. R. Gatland, R. Roy, and G. Vemuri, Phys. Rev. A 38, 5938 (1988). 